\title{
Attraction to and Avoidance of Instream Hydrokinetic Turbines by Freshwater Aquatic Organisms
}

May 2011

Prepared by

Glenn F. Cada

Mark S. Bevelhimer

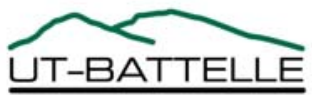




\title{
DOCUMENT AVAILABILITY
}

Reports produced after January 1, 1996, are generally available free via the U.S. Department of Energy (DOE) Information Bridge.

Web site http://www.osti.gov/bridge

Reports produced before January 1, 1996, may be purchased by members of the public from the following source.

\author{
National Technical Information Service \\ 5285 Port Royal Road \\ Springfield, VA 22161 \\ Telephone 703-605-6000 (1-800-553-6847) \\ TDD 703-487-4639 \\ Fax 703-605-6900 \\ E-mail info@ntis.gov \\ Web site http://www.ntis.gov/support/ordernowabout.htm
}

Reports are available to DOE employees, DOE contractors, Energy Technology Data Exchange (ETDE) representatives, and International Nuclear Information System (INIS) representatives from the following source.

Office of Scientific and Technical Information

P.O. Box 62

Oak Ridge, TN 37831

Telephone 865-576-8401

Fax 865-576-5728

E-mail reports@osti.gov

Web site http://www.osti.gov/contact.html

\begin{abstract}
This report was prepared as an account of work sponsored by an agency of the United States Government. Neither the United States Government nor any agency thereof, nor any of their employees, makes any warranty, express or implied, or assumes any legal liability or responsibility for the accuracy, completeness, or usefulness of any information, apparatus, product, or process disclosed, or represents that its use would not infringe privately owned rights. Reference herein to any specific commercial product, process, or service by trade name, trademark, manufacturer, or otherwise, does not necessarily constitute or imply its endorsement, recommendation, or favoring by the United States Government or any agency thereof. The views and opinions of authors expressed herein do not necessarily state or reflect those of the United States Government or any agency thereof.
\end{abstract}


Final Report

\title{
ATTRACTION TO AND AVOIDANCE OF INSTREAM HYDROKINETIC TURBINES BY FRESHWATER AQUATIC ORGANISMS
}

\author{
Glenn F. Cada \\ Mark S. Bevelhimer
}

Date Published: May 2011

\author{
Prepared for the \\ Wind and Water Power Program \\ Office of Energy Efficiency and Renewable Energy \\ U.S. Department of Energy \\ Washington, D.C
}

Prepared by

OAK RIDGE NATIONAL LABORATORY

Oak Ridge, Tennessee 37831-6283

managed by

UT-BATTELLE, LLC

for the

U.S. DEPARTMENT OF ENERGY

under contract DE-AC05-00OR22725 



\section{Table of Contents}

Page

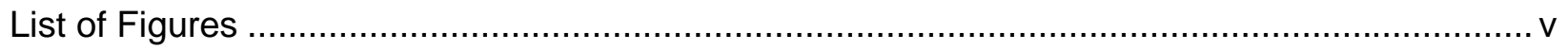

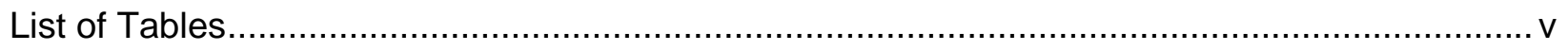

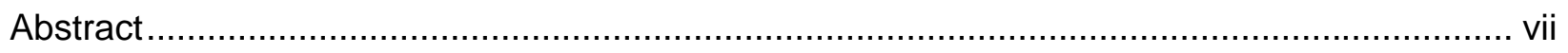

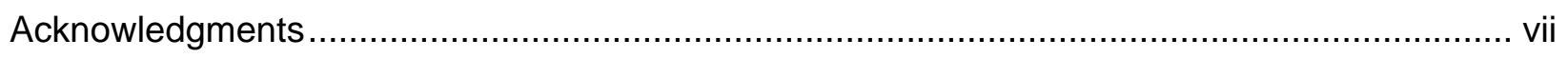

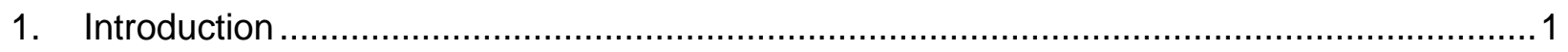

2. Proposed Hydrokinetic Energy Projects in U.S. Rivers and Estuaries ............................ 3

3. Resident and Migratory Fish Species in U.S. Rivers and Estuaries ............................. 7

4. Potential Effects on Fish Movements and Distributions....................................... 21

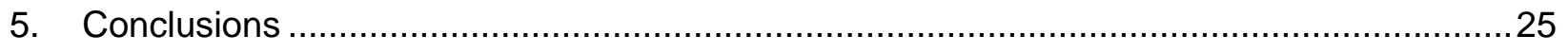

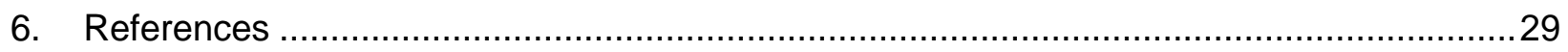





\section{List of Figures}

Figure

Page

$1 \quad$ Photographs of the Hydro Green Energy LLC horizontal axis turbine installed at the Mississippi Lock and Dam No. 2 hydroelectric project,

Hastings, Minnesota.

$2 \quad$ Preliminary permits for hydrokinetic projects issued by the Federal

Energy Regulatory Commission as of September 2010 5

3 Artist's conception of a UEK Corporations's dual hydroturbine unit, a ducted, horizontal axis hydrokinetic turbine. 6

\section{List of Tables}

Table

Page

1 Distribution and abundance of fishes in the headwaters (HW), upper (UMR) and open river (OR) segments of the Mississippi River ................................ 8

$2 \quad$ List of fishes of the Central Ohio River (River Mile $328-654$ ) ...................... 15

$3 \quad$ Juvenile fishes collected in seasonally inundated backwaters of the Atchafalaya River Basin during 2005-2006 ................................................ 18

4 Icthyoplankton collected during 2005-2006 in the Atchafalaya River Basin ........ 19 



\section{Abstract}

The development of hydrokinetic (HK) energy projects is under consideration at over 150 sites in large rivers in the United States, including the Mississippi, Ohio, Tennessee, and Atchafalaya Rivers. These waterbodies support numerous fish species that might interact with the HK projects in a variety of ways, e.g., by attraction to or avoidance of project structures. Although many fish species inhabit these rivers (about 172 species in the Mississippi River alone), not all of them will encounter the HK projects. Some species prefer low-velocity, backwater habitats rather than the high-velocity, main channel areas that would be the best sites for HK. Other, riverbank-oriented species are weak swimmers or too small to inhabit the main channel for significant periods of time. Some larger, main channel fish species are not known to be attracted to structures. Based on a consideration of habitat preferences, size/swim speed, and behavior, fish species that are most likely to be attracted to HK structures in the main channel include carps, suckers, catfish, white bass, striped bass, smallmouth bass, spotted bass, and sauger. Proper siting of the project in order to avoid sensitive fish populations, backwater and fish nursery habitat areas, and fish migration corridors will likely minimize concerns about fish attraction to or avoidance of HK structures.

\section{Acknowledgments}

We thank Jocelyn Brown-Saracino for her reviews of earlier versions of this report. This research was supported by the United States Department of Energy's (DOE) Office of Energy Efficiency and Renewable Energy, Wind and Water Power Program. Oak Ridge National Laboratory is managed by UT-Battelle, LLC, for the DOE under contract DE-AC05- 00OR22725. 



\section{Introduction}

New, renewable energy developments are being proposed for large rivers and coastal areas in the United States. It is not known whether these technologies will harm aquatic organisms, but a number of possible negative effects have been identified, some as a consequence of the new structures that would be placed in the aquatic environment. For example, hydrokinetic (HK) structures (generating devices and their associated support structures, anchors, and cables) could attract or repel animals or interfere with their movements. There is a complex relationship between structural characteristics of a river and the occurrence of fish species or age/size classes (see overview by Schlosser 1999). HK development may create new fish attraction structures, pose a threat of collision or entanglement to some organisms, and potentially alter both local movements and long distance migrations of aquatic animals. Because the transport of planktonic (drifting) life stages is affected by water velocity, localized reduction of water velocities by large, multi-unit $\mathrm{HK}$ projects could influence recruitment of some species. A variety of aquatic organisms use magnetic, chemical, and hydrodynamic cues for navigation; these have been best studied for marine organisms, but freshwater fish such as sturgeon and paddlefish also respond to these cues. Thus, in addition to mechanical obstructions, the electrical and magnetic fields and current and wave alterations produced by energy technologies could interfere with local movements or long-distance migrations. Anadromous fish (e.g., green sturgeon, salmon, steelhead, American shad) and catadromous fish (e.g., eels) migrate through both rivers and oceans and therefore may encounter both hydrokinetic devices in the rivers and wave and current energy projects in the oceans.

We compiled lists of freshwater fish species associated with large rivers for which hydrokinetic (HK) developments have been proposed (e.g., Mississippi, Ohio, Tennessee, and Atchafalaya Rivers). We divided those species into two categories: those that display only localized movements (i.e. resident fish) and those that migrate longer distances through rivers (i.e., migratory fish). We used information in the published literature to predict the behavioral responses (e.g., attraction or avoidance) of the members of these two groups to the new HK structures in rivers and estuaries. The objectives of this report are to (1) identify the potential 
interactions (attraction, avoidance, no effect) of migratory and resident aquatic organisms with HK projects; and (2) use the information to prioritize and focus studies of other environmental issues, e.g., strike, EMF, noise, and food web alterations. To accomplish this, we:

- collected information from the Federal Energy Regulatory Commission's (FERC) MHK database about the geographical distribution of proposed hydrokinetic projects, the HK turbine design (horizontal axis, vertical axis, cross-flow), the nature of the installation (attached to bridge piers or other existing structures, gravity-anchored or pile-driven into the sediments), and number of units per project.

- compared the geographical distribution of proposed projects to distribution of resident fish species and long-distance migrants in rivers and estuaries to ascertain potential interactions. This information is used to evaluate whether interference with fish movements is a significant issue for freshwater and estuarine HK projects. 


\section{Proposed Hydrokinetic Energy Projects in U.S. Rivers and Estuaries}

As of August, 2010, one license for a HK project had been issued by the FERC. The City of Hastings, Minnesota modified its license for the Mississippi Lock and Dam No. 2 Hydroelectric Project (FERC No. 4306) to incorporate two HK turbines in the tailrace of its existing dam and powerhouse on the Mississippi River. The HK array would consist of two ducted, 35-kW horizontal axis turbines provided by Hydro Green Energy LLC (Figure 1). The HK turbines would be suspended below a floating barge that is tethered to the dam and anchored for stability using anchors and pilings.

In addition to the single HK license, FERC has issued (or has pending) numerous preliminary permits for marine and HK projects. (Whereas a license authorizes construction, operation, and maintenance of a hydropower project under the FERC's jurisdiction, a preliminary permit maintains priority of application for a license at a site for up to three years while the permit holder studies project feasibility and prepares an application for license. Thus, a preliminary permit indicates the locations for potential future HK development.) Of 143 issued preliminary permits (Figure 2), 78 are for projects in the Mississippi River (mainly in Louisiana, Mississippi, and Tennessee), 19 in the Atchafalaya River in Louisiana, and 11 in the mid-Ohio River. Of 13 pending preliminary permits for riverine and estuarine areas, 4 are for projects in the mid-Ohio River, 3 in the Tennessee River, and 2 in the East River estuary in New York.

Unlike FERC licenses, preliminary permits specify the potential locations of HK projects, but do not specify in detail the turbine technology or mooring/attachment methods that might be employed. Free Flow Power Corporation (FFP) holds 86 of the 141 FERC preliminary permits, all in the lower Mississippi and Atchafalaya River basins. The FFP website (http://www.freeflow-power.com/) suggests that ducted, 3-m-diameter, horizontal-axis HK turbine-generators are being considered for these large river applications. These HK devices could be suspended from the surface, attached to bridge abutments, maintained from barges, or suspended between or attached to pylons. 

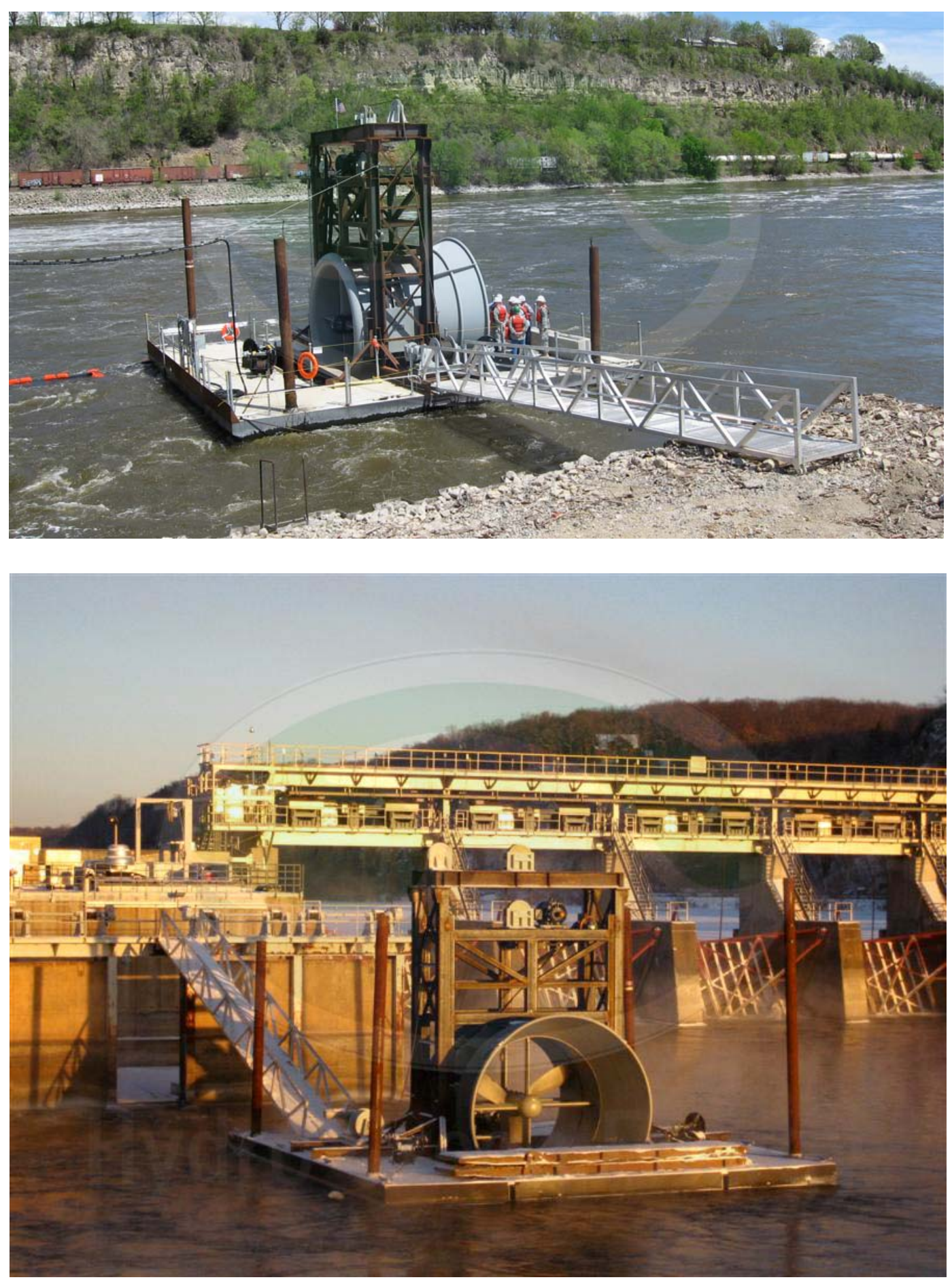

Figure 1. Photographs of the Hydro Green Energy LLC horizontal axis turbine installed at the Mississippi Lock and Dam No. 2 hydroelectric project, Hastings, Minnesota.

Source: Hydro Green Energy LLC http://www.hgenergy.com/index.html 


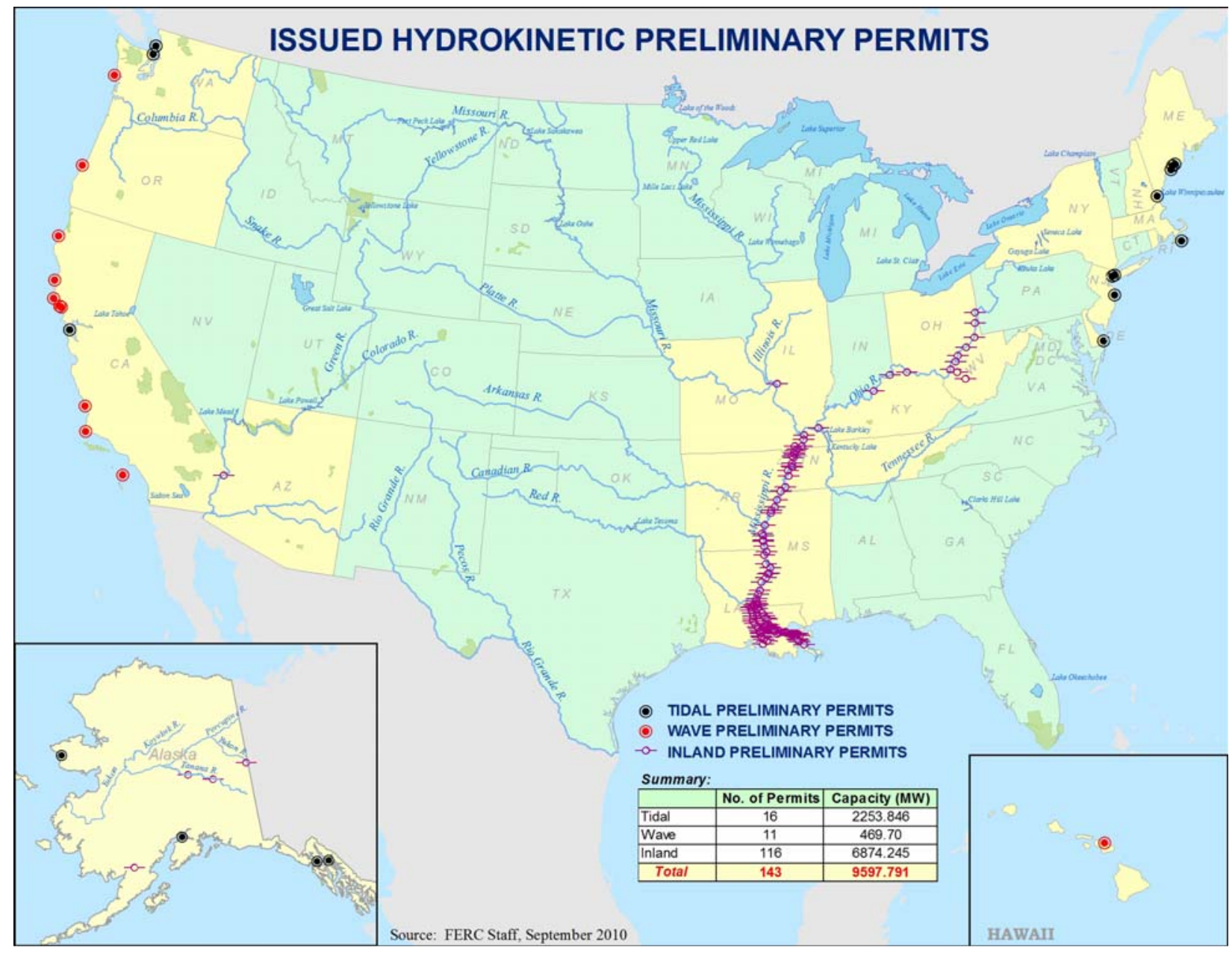

Figure 2. Preliminary permits for hydrokinetic projects issued by the Federal Energy Regulatory Commission as of September 2010. Source: http://www.ferc.gov/industries/hydropower/indus-act/hydrokinetics.asp 


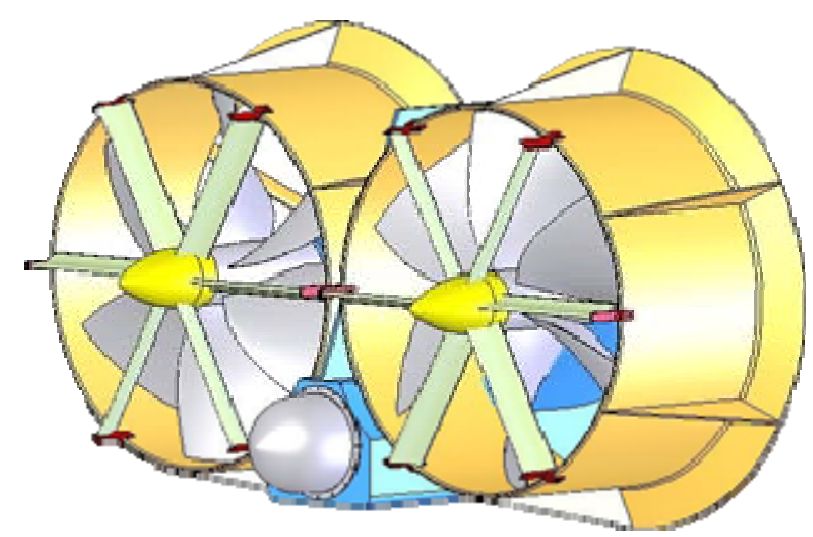

Figure 3. Artist's conception of a UEK Corporations's dual hydroturbine unit, a ducted, horizontal axis hydrokinetic turbine.

Source: UEK Corporation http://www.uekus.com/index.html

Similarly, two preliminary permits issued to UEK Corporation for sites on the Atchafalaya River are for projects that are expected to install ducted horizontal axis turbines. The fully developed 10-MW project would consist of 23 dual hydroturbine units. Each unit would be 5.2 $\mathrm{m}$ tall $\times 9.9 \mathrm{~m}$ wide $\times 6.1 \mathrm{~m}$ long (Figure 3 ). The units would be anchored in columns using common cables laid on the bottom of the river channel. The units would be located in the river channel $4.6 \mathrm{~m}$ below the surface of the water during normal or flood flows, and would be kept that that depth by an elevation control system. That is, the UEK system is a free-standing device that is maintained in the axis of the prevailing river flow by two cables secured to the main anchored bottom cables; no pilings, large concrete bases, or surface support platforms are anticipated. 


\section{Resident and Migratory Fish Species in U.S. Rivers and Estuaries}

As the largest river in North America, the Mississippi River is 3,731 km long and drains a 3.25 million square $\mathrm{km}^{2}$ watershed. Discharge in the lower river, where many HK projects are being considered, ranges from 3,568 to 55,558 m³ s (Schramm 2004). Fremling et al. (1989) listed 193 freshwater fish species in 27 families for the Mississippi River. Schramm (2004) updated that information and concluded that 172 species presently reside in the Mississippi River (see Table 1). Of these, 17 species are considered to be riverine dependent, that is, they require flowing water and sand, gravel, or rock substrate during at least some portion of their life cycle. This type of habitat is found in the main channel or channel borders, where HK projects are likely to be located. Schramm considered 31 species to prefer main channel habitat and another 77 species to prefer the channel border between the main channel and the riverbank. These main channel and channel border species would be most susceptible to interactions with HK projects. Many of the other species listed in Table 1 have more general requirements or prefer backwater habitats with no current and a soft sediment or vegetated bottom.

Although the list of fish species in the Mississippi River basin is large, a few species numerically dominate the fish community. For example, Barko et al. (2004a) reported that in the upper Mississippi River, $50 \%$ of the adult fish assemblage by number is made up of 3 species: gizzard shad (Dorosoma cepedianum), common carp (Cyprinus carpio), and channel catfish (Ictalurus punctatus). Among youngof-the-year fish, freshwater drum (Aplodinotus grunniens) and gizzard shad accounted for $76 \%$ of the total catch.

The mainstem of the Ohio River is $1579 \mathrm{~km}$ long, drains a $371,793-$ square $\mathrm{km}^{2}$ watershed, and is the second largest river in the U.S. as measured by annual discharge. The Ohio River drainage contains at least 350 species of fish (Table 2) ranging from endemic darters and dace in the headwaters to a suite of large river fish (e.g., paddlefish, blue sucker, lake sturgeon, and shovelnose sturgeon) and more than 120 mussel species, including a number that are federally listed threatened or endangered species. These figures approach half of the freshwater fish species and over a third of all mussel species found in the United States. 
Table 1. Distribution and abundance of fishes in the headwaters (HW), upper (UMR) and open river (OR) segments of the Mississippi River. Fish are resident in the Mississippi River unless noted otherwise (Residence).

Source: Schramm (2004).

\begin{tabular}{|c|c|c|c|c|c|c|c|}
\hline $\begin{array}{l}\text { Family } \\
\text { species }\end{array}$ & $\begin{array}{l}\text { Resi- } \\
\text { dence } 1\end{array}$ & $\mathrm{HW}^{2}$ & UMR $^{2}$ & $O R^{2}$ & $\begin{array}{c}\text { Back } \\
\text { water } \\
\text { dependent }\end{array}$ & $\begin{array}{l}\text { Riverine } \\
\text { dependent }\end{array}$ & $\begin{array}{c}\text { Probable } \\
\text { zone }^{3}\end{array}$ \\
\hline Ascipenseridae & & & & & & & \\
\hline $\begin{array}{l}\text { Lake sturgeon, Acipenser fulvescens } \\
\text { (Rafinesque) }\end{array}$ & & & $0^{4}$ & $\mathrm{R}^{4}$ & & Yes & MC, CB \\
\hline $\begin{array}{l}\text { Atlantic sturgeon, Acipenser oxyrhynchus } \\
\text { (Mitchill) }\end{array}$ & $\mathrm{D}$ & & & $\mathrm{R}^{5}$ & & & MC, CB \\
\hline $\begin{array}{l}\text { Pallid sturgeon, Scaphirhynchus albus } \\
\text { (Forbes and Richardson) }\end{array}$ & & & $\mathbf{R}$ & ० & & Yes & $\mathrm{MC}, \mathrm{CB}$ \\
\hline $\begin{array}{l}\text { Shovelnose sturgeon, Scaphirhynchus } \\
\text { platorynchus (Rafinesque) }\end{array}$ & & & o & o & & Yes & $\mathrm{MC}, \mathrm{CB}$ \\
\hline Polyodontidae & & & & & & & \\
\hline Paddlefish. Polyodon spathula (Walbaum) & & & o & ० & & Yes & MC, CB, BW \\
\hline Lepisosteidae & & & & & & & \\
\hline Alligator gar, Atractosteus spatula (Lacepede) & & & & $\mathbf{R}$ & Yes & & BW \\
\hline Spotted gar, Lepisosteus oculatus (Winchell) & & & $u$ & ० & Yes & & BW \\
\hline Longnose gar, Lepisosteus osseus (Linnaeus) & & & o & $\mathrm{c}$ & Yes & & MC, CB, BW \\
\hline $\begin{array}{l}\text { Shortnose gar, Lepisosteus platostomus } \\
\text { (Rafinesque) }\end{array}$ & & $\mathrm{H} 1$ & $\mathrm{c}$ & c & Yes & & MC, CB, BW \\
\hline Amiidae & & & & & & & \\
\hline $\begin{array}{l}\text { Bowfin, Amia calva (Linnaeus) } \\
\text { Anguillidae }\end{array}$ & & $\mathbf{R}$ & $\mathrm{c}$ & ० & Yes & & BW \\
\hline $\begin{array}{l}\text { American eel, Anguilla rostrata (Lesueur) } \\
\text { Hiodontidae }\end{array}$ & $\mathrm{D}$ & $\mathbf{R}$ & 0 & $\mathrm{u}$ & & & $\mathrm{CB}$ \\
\hline Goldeye, Hiodon alosoides (Rafinesque) & & & $\mathrm{u}$ & o & & & $\mathrm{CB}$ \\
\hline $\begin{array}{l}\text { Mooneye, Hiodon tergisus (Lesueur) } \\
\text { Clupeidae }\end{array}$ & & & o & $\mathrm{U} / \mathrm{R}$ & & & $\mathrm{CB}$ \\
\hline $\begin{array}{l}\text { Alabama shad, Alosa alabamae } \\
\text { (Jordan and Everman) }\end{array}$ & $\mathrm{D}$ & & & $\mathbf{R}$ & & & $\mathrm{MC}, \mathrm{CB}$ \\
\hline $\begin{array}{l}\text { Skipjack herring. Alosa chrysochloris } \\
\text { (Rafinesque) }\end{array}$ & & & O/R & $\mathrm{c}$ & & & MC, CB, BW \\
\hline Gizzard shad, Dorosoma cepedianum (Lesueur) & & A & A & A & Yes & & MC, CB, BW \\
\hline $\begin{array}{l}\text { Threadfin shad, Dorosoma petenense (Gū nther) } \\
\text { Salmonidae }\end{array}$ & & & $u$ & A & Yes & & CB. BW \\
\hline $\begin{array}{l}\text { Cisco, Coregonus artedi (Lesueur) } \\
\text { Umbridae }\end{array}$ & & $\mathbf{R}$ & $\mathbf{R}$ & & & & BW \\
\hline $\begin{array}{l}\text { Central mudminnow, Umbra limi (Kirtland) } \\
\text { Esocidae }\end{array}$ & & $u$ & ० & & Yes & & BW \\
\hline $\begin{array}{l}\text { Grass pickerel, Esox americanus vermiculatus } \\
\text { (Lesueur) }\end{array}$ & & & $\mathbf{R}$ & $\mathbf{R}$ & Yes & & BW \\
\hline Northern pike, Esox lucius (Linnaeus) & & o & o & & Yes & & BW \\
\hline Muskellunge, Esox masquinongy (Mitchill) & & $\mathrm{O} / \mathrm{U}$ & & & Yes & & BW \\
\hline Chain pickerel, Esox niger (Lesueur) & & & & $\mathrm{R}^{5}$ & Yes & & BW \\
\hline Cyprinidae & & & & & & & \\
\hline $\begin{array}{l}\text { Central stoneroller, Campostoma anomalum } \\
\text { (Rafinesque) }\end{array}$ & & $\mathbf{R}$ & $\mathbf{R}$ & $\mathrm{H} 2^{6}$ & & & $\mathrm{MC}, \mathrm{CB}$ \\
\hline Goldfish, Carassius auratus (Linnaeus) & 1 & & $\mathrm{u}$ & $\mathbf{R}$ & Yes & & BW \\
\hline
\end{tabular}


Table 1 (continued).

\begin{tabular}{|c|c|c|c|c|c|c|c|}
\hline $\begin{array}{l}\text { Family } \\
\text { species }\end{array}$ & $\begin{array}{c}\text { Resi- } \\
\text { dence } 1\end{array}$ & $\mathrm{HW}^{2}$ & UMR $^{2}$ & $O R^{2}$ & $\begin{array}{c}\text { Back } \\
\text { water } \\
\text { dependent }\end{array}$ & $\begin{array}{l}\text { Riverine } \\
\text { dependent }\end{array}$ & $\begin{array}{l}\text { Probable } \\
\text { zone }^{3}\end{array}$ \\
\hline $\begin{array}{l}\text { Grass carp. Ctenopharyngodon idella } \\
\text { (Valenciennes) }\end{array}$ & 1 & & $\mathrm{u}$ & $\mathrm{u}$ & & Yes & MC, CB, BW \\
\hline $\begin{array}{l}\text { Bluntface shiner, Cyprinella camura } \\
\text { (Jordan and Meek) }\end{array}$ & $\mathrm{P}$ & & & $\mathrm{H} 2$ & & & $\mathrm{CB}$ \\
\hline $\begin{array}{l}\text { Red shiner, Cyprinella lutrensis } \\
\text { (Baird and Girard) }\end{array}$ & & & o & $\mathrm{C} / \mathrm{O}$ & Yes & & CB, BW \\
\hline Spotfin shiner, Cyprinella spiloptera (Cope) & & $\mathrm{c}$ & $\mathrm{c}$ & $\mathbf{R}$ & & & CB, BW \\
\hline Blacktail shiner, Cyprinella venusta (Girard) & & & & 0 & & & CB, BW \\
\hline Steelcolor shiner, Cyprinella whipplei (Girard) & $P$ & & & $\mathbf{R}$ & & & CB. BW \\
\hline $\begin{array}{l}\text { Common carp. Cyprinus carpio (Linnaeus) } \\
\text { Gravel chub, Erimystax x-punctatus } \\
\text { (Hubbs and Crowe) }\end{array}$ & 1 & c & A & $\begin{array}{l}\mathrm{C} \\
\mathrm{R}\end{array}$ & Yes & & $\begin{array}{l}\text { CB, BW } \\
\text { CB, BW }\end{array}$ \\
\hline $\begin{array}{l}\text { Western silvery minnow, Hybognathus argyritis } \\
\text { (Girard) }\end{array}$ & & & & $\mathbf{R}$ & & & BW \\
\hline $\begin{array}{l}\text { Brassy minnow, Hybognathus hankinsoni } \\
\text { (Hubbs) }\end{array}$ & & $u$ & $\mathbf{R}$ & & & & $\mathrm{CB}$ \\
\hline Cypress minnow, Hybognathus hayi (Jordan) & & & & $\mathbf{R}$ & Yes & & BW \\
\hline $\begin{array}{l}\text { Mississippi silvery minnow, Hybognathus } \\
\text { nuchalis (Agassiz) }\end{array}$ & & & $\mathrm{U} / \mathrm{R}$ & o & Yes & & CB, BW \\
\hline Plains minnow, Hybognathus placitus (Girard) & & & & $\mathrm{U} / \mathrm{R}$ & & Yes & $\mathrm{MC}, \mathrm{CB}$ \\
\hline Clear chub, Hybopsis winchelli (Girard) & & & & $\mathrm{R}^{5}$ & & & $\mathrm{CB}$ \\
\hline $\begin{array}{l}\text { Silver carp, Hypophthaimichthys molitrix } \\
\text { (Valenciennes) }\end{array}$ & 1 & & $\mathrm{C} / \mathrm{O}$ & $\mathrm{c}$ & & & $\mathrm{CB}$ \\
\hline $\begin{array}{l}\text { Bighead carp. Hypophthalmichthys nobilis } \\
\text { (Richardson) }\end{array}$ & 1 & & 0 & o & & & $\mathrm{CB}$ \\
\hline $\begin{array}{l}\text { Striped shiner, Luxilus chrysocephalus } \\
\text { (Rafinesque) }\end{array}$ & $\mathrm{P}$ & & & $\mathbf{R}$ & & & $\mathrm{CB}$ \\
\hline Common shiner, Luxilus cornutus (Mitchill) & & $\mathrm{c}$ & O/R & & & & MC, CB, BW \\
\hline Ribbon shiner, Lythrurus fumeus (Evermann) & $P$ & & & $\mathbf{R}$ & & & BW \\
\hline Redfin shiner, Lythrurus umbratilis (Girard) & $\mathrm{P}$ & & $\mathbf{R}$ & $\mathrm{H} 2$ & & & CB. BW \\
\hline Speckled chub, Macrhybopsis aestivalis (Girard) & & & ० & $\mathrm{c}$ & & & $\mathrm{CB}$ \\
\hline Sturgeon chub, Macrhybopsis gelida (Girard) & & & & U/R & & & $\mathrm{CB}$ \\
\hline $\begin{array}{l}\text { Sicklefin chub, Macrhybopsis meeki } \\
\text { (Jordan and Everman) }\end{array}$ & & & & $\mathrm{U} / \mathrm{R}$ & & & $\mathrm{CB}$ \\
\hline Silver chub, Macrhybopsis storeriana (Kirtland) & & & $\mathrm{C} / \mathrm{O}$ & $\mathrm{C} / \mathrm{O}$ & & & CB. BW \\
\hline Pearl dace, Margariscus margarita (Cope) & & $\mathbf{R}$ & & & & & MC, CB, BW \\
\hline $\begin{array}{l}\text { Black carp, Mylopharyngodom piceus } \\
\text { (Richardson) }\end{array}$ & 1 & & & $\mathbf{R}$ & & & CB, BW \\
\hline Hornyhead chub, Nocomis biguttatus (Kirtland) & & o & $\mathbf{R}$ & & & & $\mathrm{CB}$ \\
\hline $\begin{array}{l}\text { Golden shiner, Notemigonus crysoleucas } \\
\text { (Mitchill) }\end{array}$ & & 0 & $\mathrm{C} / \mathrm{O}$ & $\mathrm{u}$ & Yes & & BW \\
\hline $\begin{array}{l}\text { Pallid shiner, Notropis amnis } \\
\text { (Hubbs and Greene) }\end{array}$ & & & $\mathbf{R}$ & & & & $\mathrm{CB}$ \\
\hline $\begin{array}{l}\text { Emerald shiner, Notropis atherinoides } \\
\text { (Rafinesque) }\end{array}$ & & A & A & A & & & Св, BW \\
\hline
\end{tabular}


Table 1 (continued).

\begin{tabular}{|c|c|c|c|c|c|c|c|}
\hline $\begin{array}{l}\text { Family } \\
\text { species }\end{array}$ & $\begin{array}{c}\text { Resi- } \\
\text { dence } 1\end{array}$ & $\mathrm{HW}^{2}$ & UMR $^{2}$ & $O R^{2}$ & $\begin{array}{c}\text { Back } \\
\text { water } \\
\text { dependent }\end{array}$ & $\begin{array}{l}\text { Riverine } \\
\text { dependent }\end{array}$ & $\begin{array}{c}\text { Probable } \\
\text { zone }^{3}\end{array}$ \\
\hline River shiner, Notropis blennius (Girard) & & & $\mathrm{c}$ & $\mathrm{C}$ & & & CB, BW \\
\hline Bigeye shiner, Notropis boops (Gilbert) & $\mathrm{P}$ & & & $\mathbf{R}$ & & & $\mathrm{CB}$ \\
\hline Ghost shiner, Notropis buchanani (Meek) & & & $\mathbf{R}$ & $\mathrm{U} / \mathrm{R}$ & Yes & & CB, BW \\
\hline Bigmouth shiner, Notropis dorsalis (Agassiz) & & $\mathrm{P}$ & 0 & O/R & $\mathbf{R}$ & & $\mathrm{CB}$ \\
\hline Blackchin shiner, Notropis heterodon (Cope) & & $\mathrm{u}$ & O/R & & Yes & & BW \\
\hline $\begin{array}{l}\text { Blacknose shiner, Notropis heterolepis } \\
\text { (Eigenmann and Eigenmann) }\end{array}$ & & $\mathrm{u}$ & $\mathbf{R}$ & & & & BW \\
\hline Spottail shiner, Notropis hudsonius (Clinton) & & $\mathrm{U}$ & $\mathrm{u}$ & $\mathrm{R}$ & & & $\mathrm{CB}$ \\
\hline Longnose shiner, Notropis longirostris (Hay) & & & & $u^{5}$ & & Yes & MC, СB \\
\hline Ozark minnow, Notropis nubilus (Forbes) & $\mathrm{P}$ & & $\mathbf{R}$ & $\mathbf{R}$ & & & $\mathrm{CB}$ \\
\hline $\begin{array}{l}\text { Chub shiner, Notropis potteri } \\
\text { (Hubbs and Bonham) }\end{array}$ & & & & $\mathbf{R}$ & & & $\mathrm{CB}$ \\
\hline Rosyface shiner, Notropis rubellus (Agassiz) & $\mathrm{P}$ & & $\mathbf{R}$ & & & & $\mathrm{CB}$ \\
\hline Silverband shiner, Notropis shumardi (Girard) & & & $\mathbf{R}$ & 0 & & & CB, BW \\
\hline Sand shiner, Notropis stramineus (Cope) & $P$ & $\mathbf{R}$ & ० & $u^{5}$ & & & $\mathrm{CB}$ \\
\hline Weed shiner, Notropis texanus (Girard) & & & 0 & $\mathrm{u}$ & Yes & & BW \\
\hline Mimic shiner, Notropis volucellus (Cope) & & $\mathbf{R}$ & $\mathrm{c}$ & o & & & CB, BW \\
\hline Channel shiner, Notropis wickliffi (Trautman) & & & $\mathrm{C} / \mathrm{O}$ & 0 & & & $\mathrm{MC}, \mathrm{CB}$ \\
\hline Pugnose minnow, Opsopoeodus emiliae (Hay) & & & ० & ० & Yes & & BW \\
\hline $\begin{array}{l}\text { Suckermouth minnow, Phenacobius mirabilis } \\
\text { (Girard) }\end{array}$ & & & $\mathbf{R}$ & $\mathbf{R}$ & & & CB. BW \\
\hline Northern redbelly dace, Phoxinus eos (Cope) & & c & & & & & $\mathrm{CB}$ \\
\hline $\begin{array}{l}\text { Southern redbelly dace, Phoxinus erythrogaster } \\
\text { (Rafinesque) }\end{array}$ & & $\mathrm{P}$ & & $\mathrm{H} 1$ & $\mathrm{H} 2$ & & $\mathrm{CB}$ \\
\hline Finescale dace, Phoxinus neogaeus (Cope) & & $\mathbf{R}$ & & & & & CB. BW \\
\hline $\begin{array}{l}\text { Bluntnose minnow, Pimephales notatus } \\
\text { (Rafinesque) }\end{array}$ & & $\mathrm{P}$ & 0 & 0 & $u$ & & BW \\
\hline $\begin{array}{l}\text { Fathead minnow, Pimephales promelas } \\
\text { Rafinesque }\end{array}$ & & $\mathrm{C} / \mathrm{U}$ & $u$ & $\mathbf{R}$ & Yes & & BW \\
\hline $\begin{array}{l}\text { Bullhead minnow, Pimephales vigilax } \\
\text { (Baird and Girard) }\end{array}$ & & $\mathbf{R}$ & o & 0 & Yes & & BW \\
\hline $\begin{array}{l}\text { Flathead chub, Platygobio gracilis gracilis } \\
\text { (Richardson) }\end{array}$ & & & & $\mathbf{R}$ & & Yes & $\mathrm{CB}$ \\
\hline $\begin{array}{l}\text { Eastern blacknose dace, Rhinichthys atratulus } \\
\text { (Hermann) }\end{array}$ & $\mathrm{P}$ & $u$ & $\mathbf{R}$ & & & Yes & $\mathrm{CB}$ \\
\hline $\begin{array}{l}\text { Longnose dace, Rhinichthys cataractae } \\
\text { (Valenciennes) }\end{array}$ & & $\mathrm{C} / \mathrm{O}$ & $\mathbf{R}$ & & & Yes & $\mathrm{CB}$ \\
\hline $\begin{array}{l}\text { Creek chub, Semotilus atromaculatus (Mitchill) } \\
\text { Catostomidae }\end{array}$ & & 0 & $\mathbf{R}$ & & & Yes & $\mathrm{MC}, \mathrm{CB}$ \\
\hline $\begin{array}{l}\text { River carpsucker, Carpiodes carpio } \\
\text { (Rafinesque) }\end{array}$ & & & c & A & & Yes & CB, BW \\
\hline Quillback, Carpiodes cyprinus (Lesueur) & & $\mathrm{R}$ & $\mathrm{c}$ & $\mathrm{u}$ & & & CB. BW \\
\hline $\begin{array}{l}\text { Highfin carpsucker, Carpiodes velifer } \\
\text { (Rafinesque) }\end{array}$ & & & O/U & $\mathbf{R}$ & & & CB. BW \\
\hline $\begin{array}{l}\text { White sucker, Catostomus commersoni } \\
\text { (Lacepū de) }\end{array}$ & & $\mathrm{C}$ & $\mathrm{c}$ & & & & $\mathrm{MC}, \mathrm{CB}, \mathrm{BW}$ \\
\hline
\end{tabular}


Table 1 (continued).

\begin{tabular}{|c|c|c|c|c|c|c|c|}
\hline $\begin{array}{l}\text { Family } \\
\text { species }\end{array}$ & $\begin{array}{l}\text { Resi- } \\
\text { dence }^{1}\end{array}$ & $\mathrm{HW}^{2}$ & UMR $^{2}$ & $O R^{2}$ & $\begin{array}{c}\text { Back } \\
\text { water } \\
\text { dependent }\end{array}$ & $\begin{array}{l}\text { Riverine } \\
\text { dependent }\end{array}$ & $\begin{array}{l}\text { Probable } \\
\text { zone }^{3}\end{array}$ \\
\hline Blue sucker, Cycleptus elongatus (Lesueur) & & & 0 & 0 & & Yes & $\mathrm{MC}, \mathrm{CB}$ \\
\hline Creek chubsucker, Erimyzon ablongus (Mitchill) & & & & $\mathrm{u}$ & & & BW \\
\hline $\begin{array}{l}\text { Lake chubsucker, Erimyzon succetta } \\
\text { (Lacepū de) }\end{array}$ & & & & $\mathrm{u}$ & & & BW \\
\hline $\begin{array}{l}\text { Northern hog sucker, Hypentelium nigricans } \\
\text { (Lesueur) }\end{array}$ & & 0 & $\mathbf{R}$ & & & & $\mathrm{CB}$ \\
\hline $\begin{array}{l}\text { Smallmouth buffalo, lctiobus bubalus } \\
\text { (Rafinesque) }\end{array}$ & & & $\mathrm{C} / \mathrm{O}$ & $\mathrm{A} / \mathrm{C}$ & Yes & & $\mathrm{MC}, \mathrm{CB}, \mathrm{BW}$ \\
\hline $\begin{array}{l}\text { Bigmouth buffalo, Ictiobus cyprinellus } \\
\text { (Valenciennes) }\end{array}$ & & 0 & $\mathrm{c}$ & $\mathrm{C} / \mathrm{O}$ & Yes & & CB, BW \\
\hline Black buffalo, Ictiobus niger (Rafinesque) & & & U/R & $\mathrm{u}$ & Yes & & CB, BW \\
\hline $\begin{array}{l}\text { Spotted sucker, Minytrema melanops } \\
\text { (Rafinesque) }\end{array}$ & & & $\mathrm{C} / \mathrm{O}$ & $U / R^{5}$ & Yes & & CB, BW \\
\hline $\begin{array}{l}\text { Silver redhorse, Moxostoma anisurum } \\
\text { (Rafinesque) }\end{array}$ & & 0 & $\mathrm{C} / \mathrm{O}$ & $\mathrm{H} 2$ & & & CB, BW \\
\hline River redhorse, Moxostoma carinatum (Cope) & & & O/R & $\mathbf{R}$ & & & $\mathrm{CB}$ \\
\hline $\begin{array}{l}\text { Golden redhorse, Moxostoma erythrurum } \\
\text { (Rafinesque) }\end{array}$ & & & 0 & & & & $\mathrm{MC}, \mathrm{CB}$ \\
\hline $\begin{array}{l}\text { Shorthead redhorse, Moxostoma macrole } \\
\text { pidotum (Lesueur) }\end{array}$ & & $\mathrm{c}$ & $\mathrm{C} / \mathrm{O}$ & $u^{7}$ & & & $\mathrm{MC}, \mathrm{CB}$ \\
\hline $\begin{array}{l}\text { Greater redhorse, Moxostoma valenciennesi } \\
\text { (Jordan) } \\
\text { Ictaluridae }\end{array}$ & & 0 & $\mathbf{R}$ & & & Yes & $\mathrm{MC}, \mathrm{CB}, \mathrm{BW}$ \\
\hline White catfish, Ameiurus catus (Linnaeus) & $\mathrm{P}$ & & & $\mathrm{H} 3$ & & & \\
\hline Black bullhead, Ameiurus melas (Rafinesque) & & $\mathbf{R}$ & 0 & $u$ & Yes & & BW \\
\hline Yellow bullhead, Ameiurus natalis (Lesueur) & & $\mathbf{R}$ & 0 & $\mathrm{u}$ & Yes & & BW \\
\hline Brown bullhead, Ameiurus nebulosus (Lesueur) & & $\mathbf{R}$ & 0 & & Yes & & BW \\
\hline Blue catfish, Ictalurus furcatus (Lesueur) & & & 0 & A & & & MC. CB \\
\hline $\begin{array}{l}\text { Channel catfish, lctalurus punctatus } \\
\text { (Rafinesque) }\end{array}$ & & 0 & $\mathrm{c}$ & $\mathrm{c}$ & & & CB, BW \\
\hline Mountain madtom, Noturus eleutherus (Jordan) & & & & $\mathrm{H} 1$ & & Yes & $\mathrm{CB}$ \\
\hline Stonecat, Noturus flavus (Rafinesque) & & $\mathbf{R}$ & $\mathbf{R}$ & 0 & & Yes & $\mathrm{CB}$ \\
\hline Tadpole madtom, Noturus gyrinus (Mitchill) & & $\mathbf{R}$ & 0 & $\mathrm{U} / \mathrm{R}$ & Yes & & BW \\
\hline $\begin{array}{l}\text { Freckled madtom, Noturus nocturnus } \\
\text { (Jordan and Gilbert) }\end{array}$ & & & $\mathbf{R}$ & $\mathrm{O} / \mathrm{U}$ & & & BW \\
\hline Northern madtom, Noturus stigmosus (Taylor) & & & & $\mathrm{H} 2$ & & & CB, BW \\
\hline $\begin{array}{l}\text { Flathead catfish, Pylodictis olivaris (Rafinesque) } \\
\text { Aphredoderidae }\end{array}$ & & $\mathbf{R}$ & $\mathrm{ClO}$ & A & & & $\mathrm{MC}, \mathrm{CB}$ \\
\hline $\begin{array}{l}\text { Western pirate perch, Aphredoderus sayanus } \\
\text { (Gilliams) }\end{array}$ & & & $\mathbf{R}$ & $\mathbf{R}$ & Yes & & BW \\
\hline Percopsidae & & & & & & & \\
\hline $\begin{array}{l}\text { Trout-perch, Percopsis omiscomaycus } \\
\text { (Walbaum) }\end{array}$ & & 0 & 0 & & Yes & & BW \\
\hline Gadidae & & & & & & & \\
\hline Burbot, Lota lota (Linnaeus) & & 0 & $\mathbf{R}$ & & & & CB, BW \\
\hline
\end{tabular}


Table 1 (continued).

\begin{tabular}{|c|c|c|c|c|c|c|c|}
\hline $\begin{array}{l}\text { Family } \\
\text { species }\end{array}$ & $\begin{array}{l}\text { Resi- } \\
\text { dence } 1\end{array}$ & $\mathrm{HW}^{2}$ & UMR $^{2}$ & $O R^{2}$ & $\begin{array}{c}\text { Back } \\
\text { water } \\
\text { dependent }\end{array}$ & $\begin{array}{l}\text { Riverine } \\
\text { dependent }\end{array}$ & $\begin{array}{c}\text { Probable } \\
\text { zone }^{3}\end{array}$ \\
\hline \multicolumn{8}{|l|}{ Fundulidae } \\
\hline $\begin{array}{l}\text { Golden topminnow, Fundulus chrysotus } \\
\text { (Gü nther) }\end{array}$ & $\mathrm{P}$ & & & $\mathbf{R}$ & Yes & & BW \\
\hline Banded killifish. Fundulus diaphanus (Le Sueur) & & $\mathbf{R}$ & $\mathrm{H} 1$ & & & & \\
\hline Starhead topminnow, Fundulus dispar (Agassiz) & $\mathrm{P}$ & & $\mathbf{R}$ & $\mathbf{R}$ & & & BW \\
\hline $\begin{array}{l}\text { Blackstripe topminnow, Fundulus notatus } \\
\text { (Rafinesque) }\end{array}$ & & & 0 & o & Yes & & BW \\
\hline $\begin{array}{l}\text { Blackspotted topminnow, Fundulus olivaceus } \\
\text { (Storer) }\end{array}$ & & & & 0 & Yes & & BW \\
\hline Poeciliidae & & & & & & & \\
\hline $\begin{array}{l}\text { Western mosquitofish, Gambusia affinis } \\
\text { (Baird and Girard) }\end{array}$ & & & 0 & O & Yes & & BW \\
\hline Atherinidae & & & & & & & \\
\hline Brook silverside, Labidesthes sicculus (Cope) & & 0 & $\mathrm{C} / \mathrm{O}$ & $\mathrm{C} / \mathrm{O}$ & & & BW \\
\hline Inland silverside, Menidia beryllina (Cope) & & & & 0 & & & CB, BW \\
\hline Gasterosteidae & & & & & & & \\
\hline Brook stickleback, Culaea inconstans (Kirtland) & & $\mathbf{R}$ & $\mathbf{R}$ & & & & $\mathrm{MC}, \mathrm{CB}$ \\
\hline Cottidae & & & & & & & \\
\hline Mottled sculpin, Cottus bairdi (Girard) & & $\mathbf{R}$ & & & & & \\
\hline Percichthyidae & & & & & & & \\
\hline White bass, Morone chrysops (Rafinesque) & & $\mathbf{R}$ & C & $\mathrm{C}$ & & & CB, BW \\
\hline $\begin{array}{l}\text { Yellow bass, Morone mississippiensis } \\
\text { (Jordan and Everman) }\end{array}$ & & & $\mathrm{R} / \mathrm{O}$ & 0 & & & BW \\
\hline $\begin{array}{l}\text { Striped bass, Morone saxatilis (Walbaum) }{ }^{7} \\
\text { Centrarchidae }\end{array}$ & D & & & 0 & & & $\mathrm{MC}, \mathrm{CB}$ \\
\hline Rock bass, Ambloplites rupestris (Rafinesque) & & c & $\mathrm{C} / \mathrm{O}$ & & Yes & & BW \\
\hline Shadow bass, Ambloplites arriomus (Viosca) & $\mathrm{P}$ & & & $u^{5}$ & & & BW \\
\hline Flier, Centrarchus macropterus (Lacepü de) & & & & 0 & Yes & & BW \\
\hline $\begin{array}{l}\text { Banded pygmy sunfish, Elassoma zonatum } \\
\text { (Jordan) }\end{array}$ & & & & $\mathrm{R}^{5}$ & Yes & & BW \\
\hline Green sunfish, Lepomis cyanellus (Rafinesque) & & $\mathbf{R}$ & $\mathrm{C} / \mathrm{O}$ & $\mathrm{u}$ & Yes & & BW \\
\hline Pumpkinseed, Lepomis gibbosus (Linnaeus) & & $\mathbf{R}$ & $\mathrm{C} / \mathrm{O}$ & & Yes & & BW \\
\hline Warmouth, Lepomis gulosus (Cuvier) & & & $\mathrm{O} / \mathrm{U}$ & $\mathrm{C} / \mathrm{O}$ & Yes & & BW \\
\hline Orangespotted sunfish Lepomis humilis (Girard) & & & 0 & 0 & Yes & & BW \\
\hline Bluegill, Lepomis macrochirus (Rafinesque) & & 0 & A & c & Yes & & BW \\
\hline $\begin{array}{l}\text { Longear sunfish, Lepomis megalotis } \\
\text { (Rafinesque) }\end{array}$ & & & & $u$ & Yes & & BW \\
\hline Redear sunfish, Lepomis microlophus (Gü nther) & & & & $u$ & Yes & & BW \\
\hline Bantam sunfish, Lepomis symmetricus (Forbes) & & & & $0^{5}$ & Yes & & BW \\
\hline $\begin{array}{l}\text { Smallmouth bass, Micropterus dolomieu } \\
\text { (Lacepū de) }\end{array}$ & & $\mathrm{c}$ & 0 & & & & CB, BW \\
\hline $\begin{array}{l}\text { Spotted bass, Micropterus punctulatus } \\
\text { (Rafinesque) }\end{array}$ & $\mathrm{P}$ & & & $\mathbf{R}$ & & & CB, BW \\
\hline $\begin{array}{l}\text { Largemouth bass, Micropterus salmoides } \\
\text { (Lacepū de) }\end{array}$ & & 0 & $\mathrm{c}$ & $\mathrm{c}$ & Yes & & BW \\
\hline White crappie, Pomoxis annularis (Rafinesque) & & $\mathbf{R}$ & $\mathrm{c}$ & $\mathrm{C}$ & Yes & & BW \\
\hline $\begin{array}{l}\text { Black crappie, Pomoxis nigromaculatus } \\
\text { (Lesueur) }\end{array}$ & & 0 & $\mathrm{c}$ & $\mathrm{O} / \mathrm{U}$ & Yes & & BW \\
\hline
\end{tabular}


Table 1 (continued).

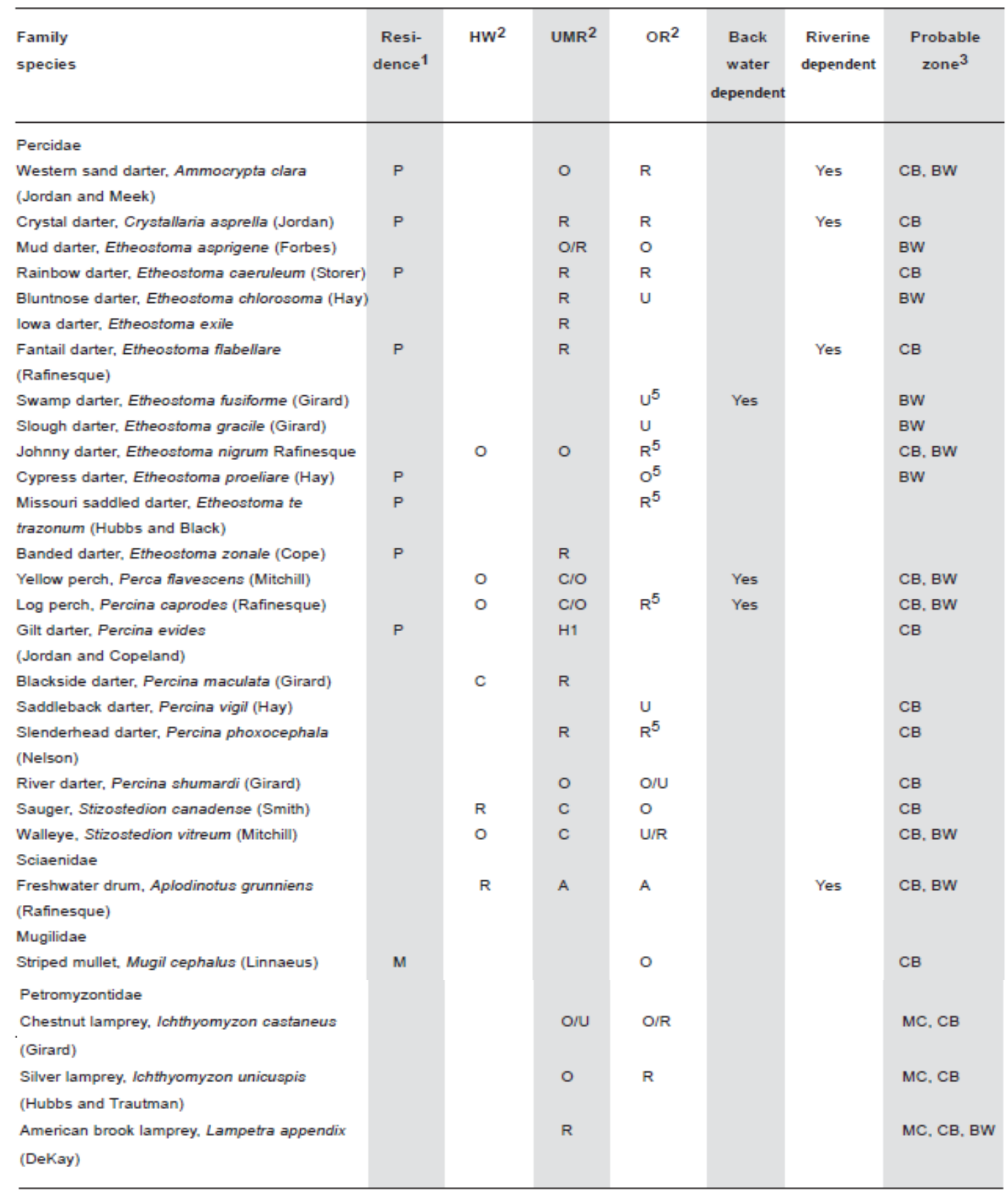




\section{Table 1 (continued).}

${ }^{1}$ All fish in this table are considered residents unless designated with one of the following letters: D - Diadromous; I - Introduced; $\mathrm{M}$ - Marine; P - Peripheral (typically occupies tributary streams and rivers but may temporarily enter the Mississippi River).

${ }^{2} \mathrm{~A}$ - Abundant in all river surveys. C - Common in most surveys. O - Occasionally collected; not generally distributed but local concentrations may occur. U - Uncommon, does not usually appear in survey samples. R - Rare. H1 - Taxon has been collected in the Mississippi River but no records of collection since 1978 (Fremling et al. 1989). H2 - Taxon reported as present by Warren et al. (2000) but abundance not known. H3 - Taxon presumed by Warren et al. (2000) to be present but not verified by collection records.

${ }^{3} \mathrm{MC}$ - Main Channel is the portion of the river that contains the thalweg and the navigation channel; water is relatively deep and the current, although varying temporally and spatially, is persistent and relatively strong. CB - Channel Border is the zone from the main channel to the riverbank. Compared to $\mathrm{MC}$, the $\mathrm{CB}$ is a zone of slower current, shallower water, and greater habitat heterogeneity. The channel border includes secondary channels and sloughs, islands and their associated sandbars, dikes and dike pools, and natural and revetted banks. BW - Backwater zone includes lentic habitats lateral to the channel border that are connected to the river for at least some time in most years. The backwater zone includes abandoned channels (including floodplain lakes) severed from the river at the upstream or both ends, lakes lateral to the channel border, ephemeral floodplain ponds, borrow pits created when levees were built, and the floodplain itself during overbank stages.

${ }^{4}$ Occasional occurrence in UMR; rare occurrence in OR attributed to stocking.

${ }^{5}$ Not listed as present in the open-river reach of the Mississippi River by Warren et al. (2000).

${ }^{6}$ Warren et al. (2000) list Mississippi stoneroller (C. a. pullum) as present in the open-river reach of the Mississippi River.

${ }^{7}$ Warren et al. (2000) list Pealip redhorse (M. m. pisolabrum) as present in the open-river reach of the Mississippi River. 
Table 2. List of fishes of the Central Ohio River (River Mile 328 - 654). Source: http://wwww.fallsoftheohio.org/OhioRiverFishList.html

Bass - Crappie Family

Largemouth Bass

Rock Bass

Smallmouth Bass

Spotted Bass

Striped Bass

White Bass

Yellow Bass

Black Crappie

White Crappie

Bowfin

Bowfin

Carp Family

Bigmouth Buffalo

Black Buffalo

Smallmouth Buffalo

Bighead Carp

$\underline{\text { Carp }}$

Grass Carp

Silver Carp

River Carpsucker

Lake Chubsucker

Goldfish

Northern Hogsucker

Quillback

Black Redhorse

Golden Redhorse

Greater Redhorse

River Redhorse

Shortnose Redhorse

Silver Redhorse

Blue Sucker

Highfin Sucker

Spotted Sucker

White Sucker

\section{Catfish Family}

Black Bullhead

Brown Bullhead

Yellow Bullhead

Blue Catfish

Channel Catfish

Flathead Catfish

White Catfish

Brindled Madtom

Mountain Madtom

Tadpole Madtom

Stonecat
Micropterus salmoides

Ambloplites rupestris

Micropterus dolomieui

Micropterus punctulatus

Morone saxatilis $^{*}$

Morone chrysops

Morone mississippiensis

Pomoxis nigromaculatus

$\underline{\text { Pomoxis annularis }}$

Amia calva

Ictiobus cyprinellus

Ictiobus niger

Hypophthalmichthys nobilis

Cyprinus carpo

Ctenopharyngodon idella

Hypophthalmichthys molitrix

Carpiodes carpio $\mathrm{t}$

Erimyzon sucetta

Carassisus auratus*

Hypentelium nigricans

Carpiodes cyprinus

Moxostoma duquesnei $\mathrm{t}$

Moxostoma erythrurum

Moxostoma valenciennesi

Moxostoma carinatum

Moxostoma macrolepidotum

Moxostoma anisurum $\mathrm{t}$

Cycleptus elongatus $\mathrm{t}$

Carpiodes velifer $\mathrm{t}$

Minytrema melanops $\mathrm{t}$

Catostomus commersoni

Icatulurus melas

Icatulurus nebulosus

Icatulurus natalis

Icatulurus furcatus

Icatulurus punctatus $\mathrm{t}$

Pylodictis olivaris $\mathrm{t}$

Icatulurus catus

Noturus miurus

Noturus eleuthurus

Noturus gyrinus

Noturus flavus $\mathrm{t}$

\section{Codfish}

American Burbot Lota lota

\section{Darters}

Banded Darter Etheostoma zonale

Crystal Darter Ammocrypta asperella

Dusky Darter Percina sciera

Eastern Sand Darter Ammocrypta pellucida

Fantail Darter

Greenside Darter

Johnny Darter

Etheostoma flabellare $\mathrm{t}$

Etheostoma blennioides $\mathrm{t}$

Etheostoma nigrum

Orangethroat Darter Etheostoma spectabile

Rainbow Darter Etheostoma caeruleum

River Darter Percina shumardi

Slenderhead Darter Percina phoxocephala

Stripetail Darter Etheostoma kennicotti

Variegate Darter Etheostoma variatum

Drum

Freshwater Drum Aplodinotus grunniens t

Eel

American Eel $\quad$ Anguilla rostrata

Gar

Alligator Gar Lepisosteus spatula

Longnose Gar Lepisosteus osseus

Shortnose Gar Lepisosteus platostomus $\mathrm{t}$

Spotted Gar Lepisosteus oculatus

Minnow-like: Chubs, Minnows and Shiners

Bigeye Chub

Cheek Chub

Hornyhead Chub

River Chub

Silver Chub

Speckled Chub

Streamline Chub

Blacknose Dace

Redside Dace

Bluntnose Minnow

Bullhead Minnow

Fathead Minnow

Silverjaw Minnow

Silvery Minnow

Suckermouth Minnow

Bigeye Shiner

Common Shiner

Emerald Shiner

Ghost Shiner

Golden Shiner

Mimic Shiner
Hybopsis amblops $\mathrm{t}$

Semotilus atromaculatus

Nocomis biguttatus

Nocomis micropogon

Hybopsis storeriana

Hybopsis aestivalis

Hybopsis dissimilis

Rhinichthys atraculatus

Clinostomus elongatus

Pimephales notatus $\mathrm{t}$

Pimephales vigilax

Pimephales promelas

Ericymba buccata

Hybognathus nuchalis

Phenacobius mirabilis

Notropis boops

Notropis cornutus

Notropis atherinoides

Notropis buchanani

Notemigonus crysoleucas

Notropis volucellus 
Ribbon Shiner

River Shiner

Rosefin Shiner

Rosyface Shiner

Sand Shiner

Silver Shiner

Spotfin Shiner

Spottail Shiner

Steelcolor Shiner

Striped Shiner

\section{Miscellaneous Minnow-type Fish}

Common Stoneroller

Blackstripe Topminnow

Brook Silverside

Mosquito Fish

Pirateperch

Troutperch

Campostoma anomolum

Fundulus notatus

Labidethes sicculus

Gambusia affinis

Aphredoderus sayanus

Percopsis omiscomaycus

\section{Lamprey}

American Brook Lamprey

Ohio Lamprey

Silver Lamprey

\section{Mooneyes}

Goldeye

Mooneye

Paddlefish

$\underline{\text { Paddlefish }}$

Perch

Logperch

Yellow Perch
Lampetra appendix Ichthyomyzon bdellium Ichthyomyzon unicuspis

Hiodon alosoides $\mathrm{t}$ Hiodon tergisus $\mathrm{t}$

Polyodon spathula

Percina caprodes $\mathrm{t}$

Perca flavescens
Pike Group

Muskellunge

Grass Pickerel

Northern Pike

Esox masquinongy

Esox americanus vermiculatus

Esox lucius*

Sauger - Walleye

Sauger

Walleye

Stizostedion canadense

Stizostedion vitreum vitreum

Sculpin

Mottled Sculpin

$\underline{\text { Cottus carolinae }}$

\section{Shad Family}

Alabama Shad

Alewife

Skipjack Shad

American Shad

American Gizzard Shad

Threadfin Shad

\author{
Alosa alabamae \\ Alosa pseudoharengus* \\ Alosa chrysochloris t \\ Alosa sapidissima* \\ Dorosoma cepedianum \\ Dorosoma petense
}

\section{Sturgeon}

Lake Sturgeon

Acipenser fulvescens

Shovelnose Sturgeon Scaphirohynchus platorhychus

\section{Sunfish}

Bluegill

Pumpkinseed

Green Sunfish

Lepomis macrochirus

Longear Sunfish

is gibbosus

Orangespotted Sunfish Lepomis humilis

Redear Sunfish Lepomis microlophus

Warmouth Lepomis gulosus

Oceanic (Freshwater Tolerant)

Coho Salmon

Oncorhynchus kisutch ${ }^{\star}$

Atlantic Rainbow Smelt Osmerus mordax

Sea Trout Salmo trutta* 
The Atchafalaya River Basin covers more than $3,600 \mathrm{~km}^{2}$ of south-central Louisiana. Although it is much smaller than the other rivers that are the subject of proposed HK projects, the Atchafalaya River normally receives $30 \%$ of the combined flows from the Mississippi and Red Rivers, and can receive up to one half of the Mississippi River discharge when needed to prevent flooding (Troutman et al. 2007). Halloran (2010) identified 26 taxa of juvenile fish from seasonally inundated backwaters of the Atchafalaya River and 12 taxa of icthyoplankton (fish eggs and larvae; Tables 3 and 4), but it is likely that the total fish assemblage of the river includes all the species found in the lower Mississippi River as well as many estuarine species.

Not all of the fish species listed in Tables 1-4 are likely to encounter HK projects. For example, among the many species found in the Mississippi River (Table 1), Schramm (2004) considers 31 to utilize the main channel (where HK projects would be located), including various sturgeon, paddlefish, gar, shad, minnows, suckers and catfish. Other species are likely to be found in the channel border between the main channel and the riverbank, and thus may interact with HK projects. Many species that orient toward structures, such as members of the bass and sunfish family, are more likely to be found in low-velocity backwater habitats. Most fish in the main channel are likely to be found near the bottom, where water velocities are lower and cover is available, rather than higher in the water column where HK rotors would be sited. 
Table 3. Juvenile fishes collected in seasonally inundated backwaters of the Atchafalaya River Basin during 2005-2006.

From Halloran (2010).

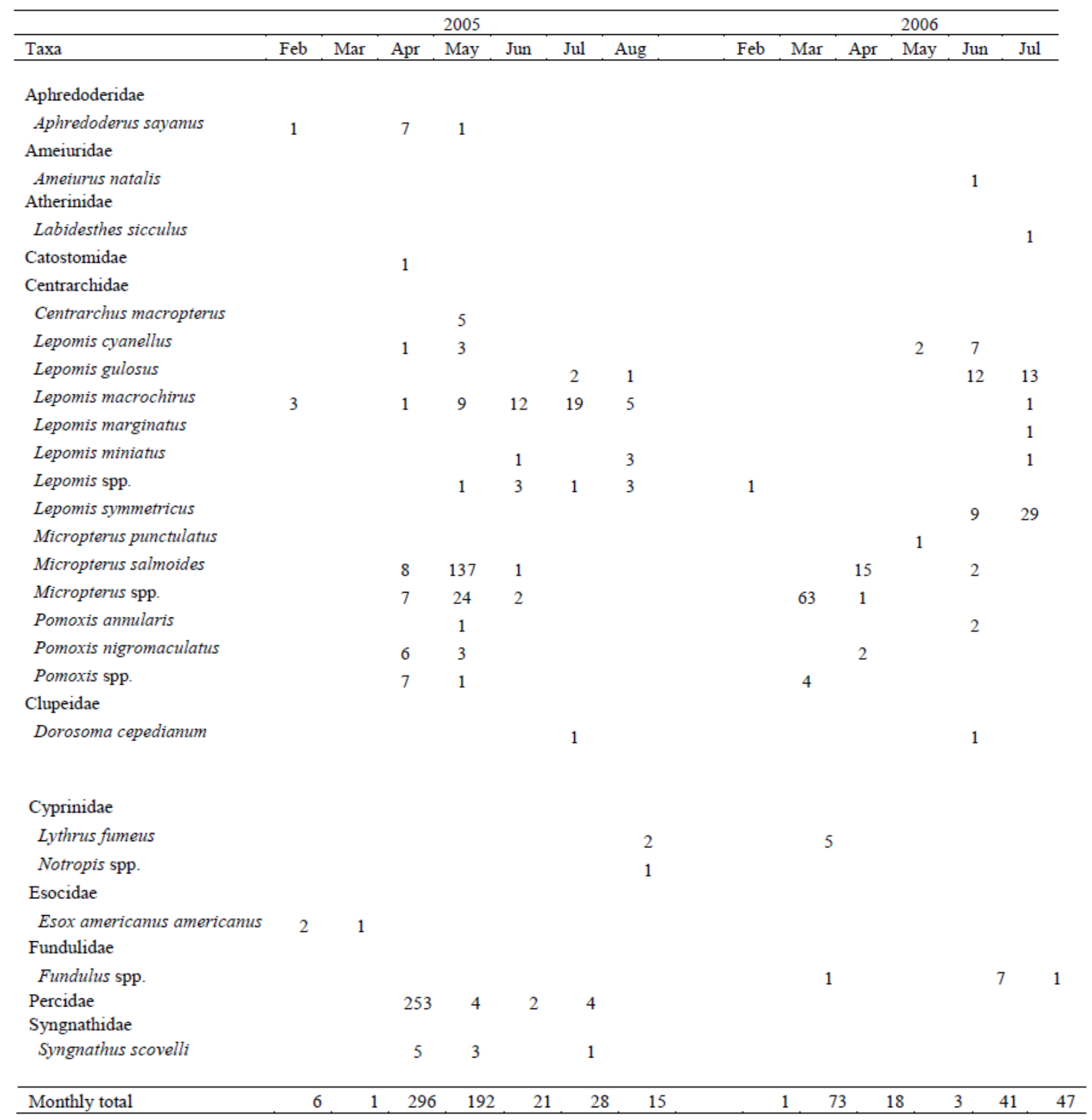


Table 4. Icthyoplankton collected during 2005-2006 in the Atchafalaya River Basin. From Halloran (2010).

\begin{tabular}{|c|c|c|c|c|c|c|c|c|c|c|c|c|c|c|c|}
\hline & & & & 2005 & & & & & & & & 2006 & & & \\
\hline Taxa & $\mathrm{Feb}$ & Mar & Apr & May & Jun & Jul & Aug & Sep & Feb & Mar & Apr & May & Jun & Jul & Aug \\
\hline \multicolumn{16}{|l|}{ Aphredoderidae } \\
\hline \multicolumn{16}{|l|}{ Aphredoderus sayamus } \\
\hline \multicolumn{16}{|l|}{ Atherinidae } \\
\hline Labidesthes sicculus & & & & & & & & & & & & & 1 & & \\
\hline Menidia beryllina & & & & 2 & & & & & & & & & & & \\
\hline Catostomidae & 1 & & 11 & 5 & 10 & & & & 18 & 21 & 20 & & & & \\
\hline \multicolumn{16}{|l|}{ Centrarchidae } \\
\hline Ambloplites spp. & & & & & & & & & 4 & & & & & & \\
\hline Lepomis spp. & 1 & & 39 & 177 & 384 & 266 & 190 & 3 & 3 & 2 & 63 & 40 & 900 & 145 & 12 \\
\hline Micropterus spp. & & 3 & & 1 & & & & & 8 & 9 & 1 & & 1 & & \\
\hline Pomoxis spp. & 2 & 6 & 38 & 1 & & & 3 & & 48 & 68 & 38 & 3 & & & \\
\hline \multicolumn{16}{|l|}{ Clupeidae } \\
\hline Dorosoma spp. & 1 & & 336 & 650 & 119 & 102 & 5 & 1 & 1 & 325 & 8365 & 680 & 3768 & 268 & 3 \\
\hline Cyprinidae & & 1 & 4 & & 21 & & & & 6 & 3 & 29 & 46 & & & \\
\hline \multicolumn{16}{|l|}{ Fundulidae } \\
\hline \multicolumn{16}{|l|}{ Fundulus spp. } \\
\hline \multicolumn{16}{|l|}{ Hidonidae } \\
\hline Hidon spp. & & & & & & & & & & & & 1 & & & \\
\hline \multicolumn{16}{|l|}{ Moronidae } \\
\hline \multicolumn{16}{|l|}{ Morone spp. } \\
\hline Percidae & & 2 & 39 & 2 & & & & & & 1 & 1 & & & & 1 \\
\hline \multicolumn{16}{|l|}{ Sciaenidae } \\
\hline Aplodinotus grunniens & & & 11 & 1 & 1 & & & & & & & 3 & 1 & & \\
\hline Unknown & & & & & & & & & & & 1 & & & & \\
\hline
\end{tabular}




\section{Potential Effects on Fish Movements and Distributions}

The numerous floating and submerged structures, mooring lines, and electrical transmission cables associated with large HK projects could interfere with the long-distance migrations of fish (e.g., juvenile and adult salmonids, paddlefish, sturgeon) if they are sited along migration corridors. Anadromous fish (e.g., green sturgeon, salmon, steelhead) and catadromous fish (e.g., eels) migrate through both rivers and oceans and therefore may encounter both hydrokinetic devices in the rivers and ocean energy projects (Dadswell et al. 1987).

Anchors and other permanent structures on the bottom will create new habitats. Artificial reefs are often constructed in marine systems in order to increase fish production, but some studies suggest that they may be less effective than natural reefs (Carr and Hixon 1997) and that they may even have deleterious effects on reef fish populations by stimulating overfishing and overexploitation (Grossman et al. 1997). In freshwater, Creque et al. (2006) studied an artificial reef constructed of granite rubble in southwestern Lake Michigan. Compared to a nearby reference site, the reef attracted more smallmouth bass (Micropterus dolomieui) and rock bass (Amblopites rupestris), but other fish species (freshwater drum, gizzard shad, yellow perch, and salmonines) were commonly found at both locations. For all of the fish, use of the artificial reef was seasonal, related to water temperature. Wills et al. (2004) examined the effects of artificial habitat structures on fish abundance in four Michigan reservoirs. Some structures (half-log habitat enhancement structures) attracted significantly greater numbers of smallmouth bass than reference areas or other artificial habitat structures. Other fish groups displayed few significant differences in abundance or nesting frequency between areas with or without structures or before and after structure placement.

The cover provided by woody debris, undercut banks, boulders, artificial structures, etc. serves three main functions: protection against predators, reduction of competition by visual isolation, and hydraulic shelter (Fausch 1993). In a review of cover for riverine fish, Allouche (2002) concluded that fish attraction to cover is largely influenced by the architectural arrangement of the cover structure (i.e., complexity, cavity space) and also the diversity of other associated habitat features generated by the structure (e.g., hydraulic heterogeneity, light 
intensity). Although the relationships between fish and cover are extremely complex, often fish diversity and abundance are increased. Habitat enhancement structures are also commonly employed in rivers to increase fish production. For example, Proboszcz and Guy (2006) found that constructed wooden half-logs were selected habitat by spotted bass (Micropterus punctulatus). Hartman and Titus (2009) reported that artificial dikes were beneficial to centrarchids (black bass and sunfish), cyprinids, and catostomids by providing velocity shelters and cover; these fish were more abundant near the dikes than at reference areas. Abundance and species richness of juvenile fishes were high near pile fields in the lower Hudson River estuary, but low under large piers (Able et al. 1998). Barko et al. (2004b) reported that wing dikes in the upper Mississippi River contained more species of adult and juvenile fishes than the nearby main channel. Among adult fish, cyprinids (minnows), clupeids (shads), and centrarchids (bass and sunfish) were generally more abundant in wing dike habitats, whereas catostomids (suckers) and some ictalurids (catfishes) were more abundant in the main channel physical habitats.

Niles and Hartman (2010) pointed out that the main channels of large rivers have swift flows, and the homogeneous habitat offers few areas of cover or low flows. High-velocity flows increase the energy that fish must expend to maintain position, and cause smaller fish, especially larvae and juveniles, to be swept downstream. Consequently, areas of low velocity like artificial dike structures provide valuable habitat in large rivers. Compared to low quality natural reference areas in the Kanawha River, WV, artificial dikes supported greater larval abundance and taxonomic richness of most fish families.

New structures in the pelagic zone of oceans and lakes (e.g., pilings or mooring cables for floating devices) will create habitat that may act as fish aggregation or attraction devices (FADs), and similar effects might occur in large rivers. These devices are extremely effective in concentrating fish and making them susceptible to harvest (Dempster and Tacquet 2004). Fish are attracted to FADs as physical structure/shelter, and they may feed on organisms attached to the structures. Artificial lighting used to distinguish structures at night may also attract aquatic organisms. If HK projects result in an aggregation of predators, they may adversely affect juvenile salmonids moving through the project area.

In marine waters, Wilhelmsson et al. (2006) found that fish abundance in the vicinity of monopiles that supported offshore wind turbines was greater than in surrounding areas, 
although species richness and diversity were similar. Most of the fish they observed near the structure were small (juvenile gobies), which may in turn attract commercially important fish looking for prey. Dempster (2005) observed considerable temporal variability in the abundance and diversity of fish associated with marine FADs moored between 3 and $10 \mathrm{~km}$ offshore. The variability was often related to the seasonal appearance of large schools of juvenile fish. Fish assemblages differed between times when predators were present or absent; few small fishes were observed near the FADs when predators were present, regardless of the season. Using FADs as an experimental tool, Nelson (2003) found that fish formed larger, more species-rich assemblages around large FADs compared to small ones, and they formed larger assemblages around FADs with fouling biota. Devices enriched with fish accumulated additional recruits more quickly than those in which fish were removed. Although there have been numerous studies of FADs in marine systems and artificial habitat structures in lakes (e.g., review of Viavant 1995), comparable information from freshwater rivers is lacking.

Fish and other aquatic organisms might also be repelled by HK projects in rivers. The visual and auditory stimuli caused by moving structures and the electromagnetic fields (EMF) associated with submerged generators and transmission cables might all conceivably invoke avoidance responses. The scientific literature relevant to possible avoidance of riverine HK projects by freshwater fish from these mechanisms is virtually non-existent, although the effects of noise and EMF are also being investigated in ongoing studies (e.g., Cada et al. 2010; Bevelhimer et al. 2010). Gurgens et al. (2000) found that paddlefish avoided 2.54-cm diameter aluminum rods in a laboratory tank, but not plastic or plastic-coated aluminum rods. The authors speculated that part of the reason why migrating paddlefish congregate below navigation locks in the Mississippi River is electrosensory aversion to the metal gates (although they conceded that high water velocities through partially opened gates might also be influential). In any case, the highly developed electrosensitivity of the paddlefish rostrum may cause them to avoid large or uncharacteristic electrical fields associated with submerged metal structures (Wilkens et al. 1997). Potentially, this sensitivity could result in avoidance of submerged pilings, generators, and electrical transmission cables associated with HK projects. If the project is large enough, fish migrations might be affected.

Regarding sound-related avoidance, it is known that fish avoid the loud, concussive noises of underwater pile driving (DOE 2009), but different species react differently even to pile- 
driving noises (Nedwell et al. 2006). The sounds from boats have been shown to cause stress in largemouth bass (Graham and Cooke 2008) and avoidance behavior in migrating salmon (Xie et al. 2008) and a variety of lake and reservoir fishes (Drastik and Kubecka 2005). In both studies, avoidance was not a problem at distances of over $10 \mathrm{~m}$. Wysocki et al. (2006) exposed freshwater fish to comparable sound pressure levels of fluctuating (discontinuous) underwater ship noises and continuous Gaussian (white) noise. Stress (measured as elevated cortisol secretion) increased in response to the ship noise but not the continuous white noise. The noise levels produced by operating HK projects have not been measured; however, the results of the Wysocki et al. (2006) experiment suggest that fish may be less affected by continuous noise produced by the HK rotors and generators than by periodic noises from boat traffic or construction. That is, fish may become habituated to a consistent noise, even though a dynamic, unpredictable noise at the same sound pressure levels may cause avoidance reactions. These types of responses to anthropogenic sounds in freshwater environments are presently being investigated (Bevelhimer et al. 2010). 


\section{Conclusions}

There have been few studies of fish attraction to artificial structures like HK in large rivers, but some useful Before-After, Control-Impact (BACl) studies of artificial reefs in freshwater reservoirs have been published (Wills et al. 2004; Creque et al. 2006). Based on these limited studies, the attraction of freshwater fish to HK structures is likely to be seasonal rather than constant; the use of the structure may be related to water temperature or seasonal movements/migrations. Attraction to HK structures will certainly be species-specific. Some freshwater fish species show little interest in mid-water structures, whereas others such as smallmouth bass are more likely to be attracted, especially to overhead cover (Creque et al. 2006). Numerous studies have shown that black bass (Micropterus spp.) and other members of the Family Centrarchidae (bass and sunfish) have an affinity for structures throughout summer and during the nesting season. But the depth at which structures are placed and the complexity of nearby natural habitats will also influence the attractiveness of artificial structures (Wills et al. 2004).

Schramm (2004) listed 172 resident, freshwater fish species in the Mississippi River. Many of these species are found in the lower segments of the river and its larger tributaries, where the bulk of the HK projects have been proposed. However, a number of these species will likely have little interaction with HK structures in the main channels of the rivers. Of the 137 species that Schramm was able to assign to preferred habitat zones, none is expected to reside in main channel habitats throughout their life cycle, 24 are expected to occupy one or more channel border habitats throughout their life cycle, and 50 species are expected to reside in one or more backwater habitats throughout their lifecycle. Those species that prefer backwater habitats (characterized by slow currents, shallow water, fine sediments and vegetation) will not normally encounter HK structures. Among the 31 main channel- and 77 channel borderoccupying species, some prefer open waters and would not necessarily be attracted to project structures. Many of the 77 channel border species are small minnows, dace, and shiners (Family Cyprinidae) that are weak swimmers and unlikely to venture into the high-velocity channel for long. Based on a consideration of habitat preferences, size/swim speed, and behavior, fish species that are most likely to be attracted to HK structures in the main channel 
include carps, suckers, catfish, white bass, striped bass, smallmouth bass, spotted bass, and sauger.

For fish in marine, lake, and reservoir systems at least, the average number of individuals and species attracted increases with the structural complexity, volume, size, and surface area of habitat enhancement structures (Wills et al. 2004). The same effect might be expected near HK projects in large rivers. However, compared to dikes and other artificial habitats that are purposely placed in rivers to support fish, HK structures are less likely to provide desirable habitat. For example, Niles and Hartman (2010) noted that incorporation of natural components (to make them attractive to a wider variety of species) and provision of low flow areas, cover, and foraging habitats are important qualities of effective habitat enhancement structures in large rivers. These are not likely to be important elements of HK project structures, and thus their attractiveness to fish will be limited. In general, the additional habitat created by HK structures may not be significant in the context of numerous other man-made structures in large rivers such as bridges, piers, docks, wing dikes, and revetments.

In the ocean, FADs attract fish because they provide food and shelter (Castro et al. 2002); subsequently, they also attract predators (Dempster 2005) that can in turn attract commercial and sport fisheries. Without well-designed monitoring, it will be difficult to determine whether an HK project will enhance populations of aquatic organisms (by providing more habitat to support more fish), will have no overall effect (because it simply draws fish from other, nearby areas), or will decrease fish populations (by facilitating harvest by predators and fishermen). Kingsford (1999) pointed out that the determination of the effects of FADs at a particular location is complicated by the influence of non-independent factors: proximity of other FADs, interconnection of multiple FADs to provide routes for the movement of associated fishes, and temporal dependence (the number of fish present at one sampling date influencing the number at the next sampling date due to fish becoming residents). He described statistical approaches that could be applied to experiments on the effects of FADs on fish populations and solutions to the independent factor problems.

Allouche (2002) noted that whether the addition of cover structures really increases the carrying capacity of a stream or simply causes a redistribution of the existing capacity still remains controversial. Compared to nearby reference areas without structures, a greater abundance and diversity of certain riverine fish species can be expected near HK structures, 
unless the fish are repelled by other aspects of the device (noise, electromagnetic fields, and rotor movements). Although a possible "FAD effect" has not been studied for HK installations in large rivers, temporary/seasonal attraction of fish to structures that are less valuable than natural habitats is unlikely to alter fish populations or aquatic communities.

Avoidance of MHK projects by fish may result from adverse stimuli presented by movements of the rotors, noise, and EMF emitted by the generating and electrical transmission cables. As with structures, the possible effects on fish of noise from and motions of the HK devices would have to be placed in the context of other such stimuli in the main channels of rivers, especially recreational and commercial boat traffic. Little is known about these effects, although the noise study of Wysocki et al. (2006) suggests that fish may be repulsed by the aperiodic, startling stimuli from boats and become habituated to constant, low-level stimuli from HK machines. Some freshwater fish are known to be sensitive to electromagnetic fields (e.g., sturgeon and paddlefish), and studies are being carried out to determine the sensitivity of these and other freshwater species to the levels of magnetic fields that are expected to be produced by HK projects (Cada et al. 2010).

\section{Monitoring Potential Interference with Animal Movements and Migrations}

Because there is insufficient information about the likely effects of structures associated with large energy conversion projects on the movements and migrations of aquatic animals, monitoring of attraction or avoidance will be needed, at least initially. With regard to the local movements, the new structures may act as FADs and increase the local abundance of fish. Changes in numbers and relative abundances of fish populations could be monitored before and after project installation, using control and impacted sites (i.e., a BACl experimental design). Determining the effects of FADs at a particular location is complicated by the influence of non-independent factors including the proximity of other FADs (i.e., other HK units), the interconnection of multiple FADs to provide routes for the movement of associated fishes, and temporal dependence (where the number of fish present at one time influences the number at the next time due to fish becoming residents). Kingsford (1999) described statistical approaches that could be applied to experiments on the effects of FADs on fish populations and solutions to the independent factor problems. 
Changes in the abundance of fish in the area of the project could be assessed with acoustic monitoring techniques. For example, Mueller et al. (2006) described the use of visual and acoustic cameras to determine fish presence, behavior, and habitat associations in rivers. Brehmer et al. (2003) were able to monitor fish aggregations, fish behaviors, diel variations, and interactions with artificial structures (vertical longlines suspended between anchors and buoys) using multi-beam side scan sonar. De Leeuw et al. (2007) reviewed techniques for assessing the fish communities of large floodplain rivers, including the use of both hydroacoustic methods and more traditional trawling and netting methods. These techniques could be adapted to the particular circumstances and sampling needs of the HK project.

Effects on long distance movements and migrations are more difficult to assess, and will depend initially on telemetry studies of animals migrating in the vicinity of the energy project. Once the species of interest are determined, active tracking of individuals marked with electronic tags may provide the most useful information about whether or not the HK project interferes with migrations. Ransom et al. (2008), Tripp and Garvey (2009), and Lindley et al. (2011) provided recent examples of the application of acoustic tag monitoring in large rivers.

\section{Mitigating Attraction/Avoidance Effects of Riverine HK Projects}

If attraction to or avoidance of HK projects proves to be an issue, the most reliable impact mitigation measure is likely to be proper siting of the energy project in order to avoid sensitive fish populations, habitat areas, and fish migration corridors. For example, it may be prudent to avoid siting the HK project near the entrance of backwaters that serve as nursery habitat for juvenile fish or backwater-dependent adult fish because this location would expose a greater number of species to project effects. Positioning the HK project in the main channel of highest water velocities and least diverse habitats would greatly reduce the amount of interaction with riverine fish. Sound insulation may be needed if noises produced by the generators repel fish or interfere with migrations. Mitigation of EMF effects from freshwater HK projects may be easier than in oceans because rivers are likely to support fewer EMF-sensitive organisms and need shorter cables to transmit electricity from the HK generator to shore. Exposure to EMF can be reduced by burying the transmission cables in the sediments, thereby increasing the separation between the source of the field and any aquatic organisms in the open water. 


\section{References}

Able, K.W., J.P. Manderson, and A.L. Studholme. 1998. The distribution of shallow water juvenile fishes in an urban estuary: The effects of manmade structures in the lower Hudson River. Estuaries 21(4B):731-744.

Allouche, S. 2002. Nature and functions of cover for riverine fish. Bull. Fr. Peche Piscic. 365/366:297-324.

Barko, V.A., M.W. Palmer, D.P. Herzog, and B.S. Ickes. 2004a. Influential environmental gradients and spatiotemporal patterns of fish assemblages in the unimpounded upper Mississippi River. American Midland Naturalist 152:369-385.

Barko, V.A., D.P. Herzog, R.A. Hrabik, and J.S. Scheibe. 2004b. Relationship among fish assemblages and main-channel-border physical habitats in the unimpounded upper Mississippi River. Transactions of the American Fisheries Society 133:371-384.

Bevelhimer, M., G. Cada, M. Johnson, and W. Bonness. 2010. Potential Effects of Noise from Hydrokinetic Devices on Aquatic Organisms. FY2010 Annual Progress Report to the Wind and Water Power Program, U.S. Department of Energy, Washington, DC. 26 p.

Brehmer, P., F. Gerlotto, J. Guillard, F. Sanguinede, Y. Guennegan, and D. Buestel. 2003. New applications of hydroacoustic methods for monitoring shallow water aquatic ecosystems: the case of mussel culture grounds. Aquatic Living Resources 16:333-338.

Cada, G., M. Bevelhimer, K. Riemer, and J. Turner. 2010. Effects on Freshwater Organisms of Magnetic Fields Associated with Instream Hydrokinetic Turbines. FY2010 Annual Progress Report to the Wind and Water Power Program, U.S. Department of Energy, Washington, DC. $35 \mathrm{p}$.

Carr, M.H. and M.A. Hixon. 1997. Artificial reefs: The importance of comparisons with natural reefs. Fisheries 22(4):28-33.

Castro, J.J., J.A. Santiago, and A.T. Santana-Ortega. 2002. A general theory on fish aggregation to floating objects: An alternative to the meeting point hypothesis. Reviews in Fish Biology and Fisheries 11:255-277.

Creque, S.M., M.J. Raffenberg, W.A. Brofka, and J.M. Dettmers. 2006. If you build it, will they come? Fish and angler use at a freshwater artificial reef. North American Journal of Fisheries Management 26:702-713. 
Dadswell, M.J., R.J. Klauda, C.M. Moffitt, R.L. Saunders, R.A. Rulifson, J.E. Cooper. 1987. Common Strategies of Anadromous and Catadromous Fishes. American Fisheries Society Symposium 1, Bethesda, MD.

De Leeuw, J.J., A.D. Buijse, G. Haidvogl, M. Lapinska, R. Noble, R. Repecka, T. Virbickas, W. Wisniewolski, and C. Wolter. 2007. Challenges in developing fish-based ecological assessment methods for large floodplain rivers. Fisheries Management and Ecology 14:483-494.

Dempster, T. and M. Taquet. 2004. Fish aggregation device (FAD) research: gaps in current knowledge and future directions for ecological studies. Reviews in Fish Biology and Fisheries $14: 21-42$.

Dempster, T. 2005. Temporal variability of pelagic fish assemblages around fish aggregation devices: biological and physical influences. Journal of Fish Biology 66:1237-1260.

DOE (U.S. Department of Energy). 2009. Report to Congress on the Potential Environmental Effects of Marine and Hydrokinetic Energy Technologies. Prepared in response to the Energy Independence and Security Act of 2007, Section 633(b). Wind and Hydropower Technologies Program, U.S. Department of Energy, Washington, DC. 89 p. + appendices.

Drastik, V. and J. Kubecka. 2005. Fish avoidance of acoustic survey boat in shallow waters. Fisheries Research 72(2005):219-228.

Fausch, K.D. 1993. Experimental analysis of microhabitat selection by juvenile steelhead (Oncorhynchus mykiss) and coho salmon (O. kisutch) in a British Columbia stream. Canadian Journal of Fisheries and Aquatic Sciences 50:1198-1207.

Fremling, C.R., J.L. Rasmussen, R.E. Sparks, S.P. Cobb, C.F. Bryan, and T.O. Claflin. 1989. Mississippi River fisheries: A case history. P. 309-351 In D.P. Dodge (ed). Proceedings of the International Large River Symposium. Department of Fisheries and Oceans, Ottawa, Canada.

Graham, A.L. and S.J. Cooke. 2008. The effects of noise disturbance from various recreational boating activities common to inland waters on the cardiac physiology of a freshwater fish, the largemouth bass (Micropterus salmoides). Aquatic Conservation: Marine and Freshwater Ecosystems 18:1315-1324.

Grossman, G.D., G.P. Jones, and W.J. Seaman, Jr. 1997. Do artificial reefs increase regional fish production? A review of existing data. Fisheries 22(4):17-23.

Gurgens, C., D.F. Russell, and L.A. Wilkens. 2000. Electrosensory avoidance of metal obstacles by the paddlefish. Journal of Fish Biology 57:277-290.

Halloran, B. T. 2010. Early life history dynamics of the fish community in the Atchafalaya River Basin. Ph.D. Dissertation, School of Renewable Natural Resources, Louisiana State University, Baton Rouge, LA. 115 p. 
Hartman, K.J. and J.L. Titus. 2009. Fish use of artificial dike structures in a navigable river. River Research and Applications (2009).

Kingsford, M.J. 1999. Fish attraction devices (FADs) and experimental designs. Scientia Marina 63(3-4):181-190.

Lindley, S.T., D.L. Erickson, M.L. Moser, G. Williams, O.P. Langness, B.W. McCovey, Jr., M. Belchik, D. Vogel, W. Pinnix, J.T. Kelly, J.C. Heublein, and A.P. Klimley. 2011. Electronic tagging of green sturgeon reveals population structure and movement among estuaries. Transactions of the American Fisheries Society 140(1):108-122.

Mueller, R.P., R.S. Brown, H . Hop, and L. Moulton. 2006. Video and acoustic camera techniques for studying fish under ice: a review and comparison. Reviews in Fish Biology and Fisheries 16:213:226.

Nedwell, J.R., A.W.H. Turnpenny, J.M. Lovell, and B. Edwards. 2006. An investigation into the effects of underwater piling noise on salmonids. Journal of the Acoustic Society of America 120(5):2550-2554.

Nelson, P.A. 2003. Marine fish assemblages associated with fish aggregating devices: Effects of fish removal, FAD size, fouling communities, and prior recruits. Fishery Bulletin 101(4):835850.

Niles, J.M. and K.J. Hartman. 2010. Temporal distribution and taxonomic composition differences of larval fish in a large navigable river: A comparison of artificial dike structures and natural habitat. River Research and Applications (2010).

Normandeau Associates, Inc. (2009). An Estimation of Survival and Injury of Fish Passed Through the Hydro Green Energy Hydrokinetic System, and a Characterization of Fish Entrainment Potential at the Mississippi Lock and Dam No. 2 Hydroelectric Project (P-4306), Hastings, Minnesota. Final Report prepared for Hydro Green Energy, LLC, Houston, Texas. 82 p.

Proboszcz, S.L. and C.S. Guy. 2006. Evaluation of habitat enhancement structure use by spotted bass. The Prairie Naturalist 38(4):223-238.

Ransom, B.H., T.W. Steig, M.A. Timko, and P.A. Nealson. 2008. Basin-wide monitoring of salmon smolts at US dams. Hydropower \& Dams 3:2-7.

Schlosser, I.J. 1991. Stream fish ecology: A landscape perspective. Bioscience 41(10):704-712.

Schramm, H.L. Jr. 2004. Status and management of Mississippi River fisheries. Available at http://mekonginfo.org/mrc Mekong River Commission. 34 p. 
Troutman, J.P., D.A. Rutherford, and W.E. Kelso. 2007. Patterns of habitat use among vegetation-dwelling littoral fishes in the Atchafalaya River Basin, Louisiana. Transactions of the American Fisheries Society 136:1063-1075.

Tripp, S. and J. Garvey. 2009. Fish Passage in the Upper Mississippi River System. Annual Report. Fisheries and Illinois Aquaculture Center, Southern Illinois University, Carbondale, IL. November 6, 2009. 95 p.

Viavant, T. 1995. Fish attraction to artificial structure in Chena Lakes, Alaska. Fishery Data Series No. 95-6, Alaska Department of Fish and Game, Anchorage, AK. 14 p.

Wilhelmsson, D., T. Malm, and M.C. Ohman. 2006. The influence of offshore windpower on demersal fish. ICES Journal of Marine Science 63:775-784.

Wilkens, L.A., D.F. Russell, X. Pei, and C. Gurgens. 1997. The paddlefish rostrum functions as an electrosensory antenna in plankton feeding. Proceedings of the Royal Society of London B. 264:1723-1729.

Wills, T.C., M.T. Bremigan, and D.B. Hayes. 2004. Variable effects of habitat enhancement structures across species and habitats in Michigan reservoirs. Transactions of the American Fisheries Society 133:399-411.

Wysocki, L.E., J.P. Dittami, and F. Ladich. 2006. Ship noise and cortisol secretion in European freshwater fishes. Biological Conservation 128:501-508.

Xie, Y., C.G.J. Michielsens, A.P. Gray, F.J. Martens, and J.L. Boffey. 2008. Observation of avoidance reactions of migrating salmon to a mobile survey vessel in a riverine environment. Canadian Journal of Fisheries and Aquatic Science 65:2178-2190. 
ORNL/TM-2011/131

\section{INTERNAL DISTRIBUTION}

1. M. S. Bevelhimer

2. G. F. Cada

3. G. K. Jacobs

4. L. Liang

5. M. J. Peterson
6. J. W. Saulsbury

7. G. P. Zimmerman

8. ORNL Office of Technical Information and Classification

\section{EXTERNAL DISTRIBUTION}

9. Jocelyn Brown-Saracino, U.S. Department of Energy, Office of Wind and Hydropower Technology Program, Forrestal Building, EE-2B, 1000 Independence Avenue, S.W., Washington, DC 20585 